\title{
Improvement of the modeling and diagnosis of hepatic encephalopathy in rats
}

\begin{abstract}
Introduction: Modeling of liver cirrhosis (LC) and hepatic encephalopathy (HE) in rats has some limitations namely high mortality of lab animals or high duration of LC formation. Diagnosis of HE was accomplished with the model of food conditioned reflex that allowed evaluating only locomotors activity of animals but not their ability to think and remember.

Aims \& Methods: That is why the aim of the study was the optimization of the HE modeling and diagnosis. Intra-peritoneal administration of $1 \mathrm{ml} / \mathrm{kg} 15 \%$ solution of $\mathrm{CCl}_{4}$ in olive oil four times a week for 4weeks has been suggested for optimal simulation of $\mathrm{HE}^{4}$ in rats. The development of HE, namely the ability of animals to memorization, learning and thinking was studied by the formation of a food conditioned reflex in the T-shaped maze. 14 rats were divided into two groups: 1 st -intact rats and 2nd-rats pretreated with $\mathrm{CCl}_{4}$. The dynamic of reducing the time of getting animals to the food reinforcement in the maze as a result of remembering the location of food was estimated during 14days.
\end{abstract}

Results: In the first day of food conditioned reflex study it was established that intact rats reached the food in $157 \pm 47 \mathrm{sec}$. Contrary, the time of getting to food of $\mathrm{CCl}_{4}$-rats was 2,1 times $(\mathrm{p}<0.05)$ longer that suggests the less activity and lethargy of these animals. In the next 7 days, there were not any changes in the time of getting to food in T-shape maze in the intact group as well as in the group pretreated with $\mathrm{CCl}_{4}$. Since the 7 th day we have shown a steady acceleration of finding the food by intact rats. However, there were not registered data about faster food reinforcement in the group of animals that got $\mathrm{CCl}_{4}$ injection. Moreover, in the group of intact rats we have established the decrease in time of finding the food by $92 \%(\mathrm{p}<0.05)$ as compared with the first day of experiment. Such changes were not obvious for the $\mathrm{CCl}_{4}$-group: we did not find the significant difference between the reaching food of $\mathrm{CCl}_{4}$-rats at the start and at the 14th day of experiment in the T-shaped maze. Thus, we registered that the time of finding the food in the $\mathrm{CCl}_{4}$ group was $21.4(\mathrm{p}<0.001)$ greater than in the intact group at the 14 th day of study in the T-shaped maze. The impairment of food conditioned reflex formation has showed lack of remembering and thinking in rats pretreated with $\mathrm{CCl}_{4}$.

Conclusion: we have updated the scheme of modeling LC and HE by the decrease of time of the LC development and the reduction of animal mortality as result of toxic action by $\mathrm{CCl}_{4}$. The improved diagnosis of $\mathrm{HE}$ was achieved by using the methodology of forming a food conditioned reflex in rats. This method can be recommended as a basic for HE modeling and can be used for study new treatment strategies of this disease.

Keywords: $\mathrm{CCl}_{4}$, liver cirrhosis, hepatic encephalopathy, food conditioned reflex

\author{
Volume 3 Issue 3 - 2017
}

\author{
Elina Manzhalii,' Falalyeyeva T,' Beregova T, ${ }^{3}$ \\ Virchenko $\mathrm{O}^{2}$ \\ 'Department of Propedeutics of Internal medicine II, \\ Bogomolets National Medical University, Ukraine \\ ${ }^{2}$ Department of Biology, Taras Shevchenko National University \\ of Kiev, Ukraine \\ ${ }^{3}$ Department of Biology, Bogomolets National Medical \\ University, Ukraine
}

Correspondence: Elina Manzhalii, Department of Propedeutics of Internal medicine II, Bogomolets National Medical University, Kiev, Ukraine, Email elinam@ukr.net

Received: November 16, 2016 | Published: July 12, 2017

\section{Introduction}

One of the challenging problem in gastroenterology is the treatment of liver cirrhosis (LC) and hepatic encephalopathy (HE) as one of its dangerous complications. ${ }^{1,2} \mathrm{HE}$ is the frequent disorder that hits approximately $50-80 \%$ of cirrhotic patients. ${ }^{3,4} \mathrm{HE}$ is a complex, reversible neuropsychiatric syndrome, complicating the course of liver disease. In recent guidelines published jointly by the European and American Associations for the Study of the Liver, HE was defined as "brain dysfunction caused by liver insufficiency or portal systemic shunting". ${ }^{5}$ Despite the fact that the syndrome was probably recognized thousands of years ago, the exact pathogenesis and diagnosis remain not enough comprehensible. ${ }^{6}$ The pathogenesis is thought to be attributable to both neuro-chemical and neuro-physiological disorders of the brain. The essentially reversible nature suggests a metabolic cause. ${ }^{6}$ This pathology is associated with progressive dystrophy of brain tissue and can lead to patients disability. ${ }^{71}$ Despite agreement between investigators that $\mathrm{HE}$ warrants further investigation and probably screening and treatment, there is little consensus as to the optimal instruments with which to diagnose and monitor the condition. This has a significant role in the discrepancy between studies, as to the prevalence of minimal HE, with quoted prevalence varying from $27 \%$ to $75 \%$ according to the battery of tests used, interpretation of these tests, and populations studied. Some studies have used just one psychometric test, whereas other studies have used up to 26 tests. In the latter case, tests needed to be spread over 2 days to avoid fatigue. ${ }^{6,3} \mathrm{HE}$ affects cognitive, effective/emotional, behavioral, and bio-regulatory domains. Each broad domain may be subdivided into various components. For example, cognition may include evaluation of psychomotor speed, visual praxis, attention, concentration, and level of consciousness. Overt or clinically apparent HE in patiens should be excluded by careful and detailed neuropsychiatric examination and anamnestic enquiry. Particular attention should be paid to cognitive and motor function, ability to perform activities of daily living, and sleep-wake cycle abnormalities. ${ }^{6}$ 


\section{Results}

Therefore, the study of the mechanisms of diagnostics and pathogenesis and possible strategies of treatment of $\mathrm{HE}$ is current issue of scientists all over the world. Today $\mathrm{CCl}_{4}$ is used for modeling of LC and $\mathrm{HE}$ in rats. However, this method has some limitations namely high mortality of lab animals or high duration of LC formation. ${ }^{8}$ Also diagnosis of HE was made on the study of the food conditioned reflex that allowed evaluating only locomotors activity of animals but not their ability to think and remember. That is why the aim of the study was the optimization of HE modeling and diagnose. The modeling of $\mathrm{HE}$ in rats with hepatitis and liver cirrhosis was improved. Considering the literature data and selecting doses, intra-peritoneal administration of $1 \mathrm{ml} / \mathrm{kg} 15 \%$ solution of $\mathrm{CCl}_{4}$ in olive oil four times a week for 4 weeks has been suggested for optimal simulation of HE in rats. The development of HE, namely the ability of animals to memorization, learning and thinking was studied by the formation of a food conditioned reflex in the T-shaped maze (Figure 1). 14 rats were divided into two groups: 1-intact rats and 2-rats pretreated with $\mathrm{CCl}_{4}$. The dynamic of reducing the time of getting animals to the food reinforcement in the maze as a result of remembering the location of food was estimated during 14days. In the first day of food conditioned reflex study it was established that intact rats reached the food in $157 \pm 47 \mathrm{sec}$ (Figure 2). Contrary, the time of getting to food of $\mathrm{CCl}_{4}$-rats was 2,1 times $(\mathrm{p}<0.05)$ longer that suggests the less activity and lethargy of these animals. In the next 7 days, there were not any changes in the time of getting to food in T-shape maze in the intact group as well as in the group pretreated with $\mathrm{CCl}_{4}$. Since the 7 th day we have shown a steady acceleration of finding the food by intact rats.

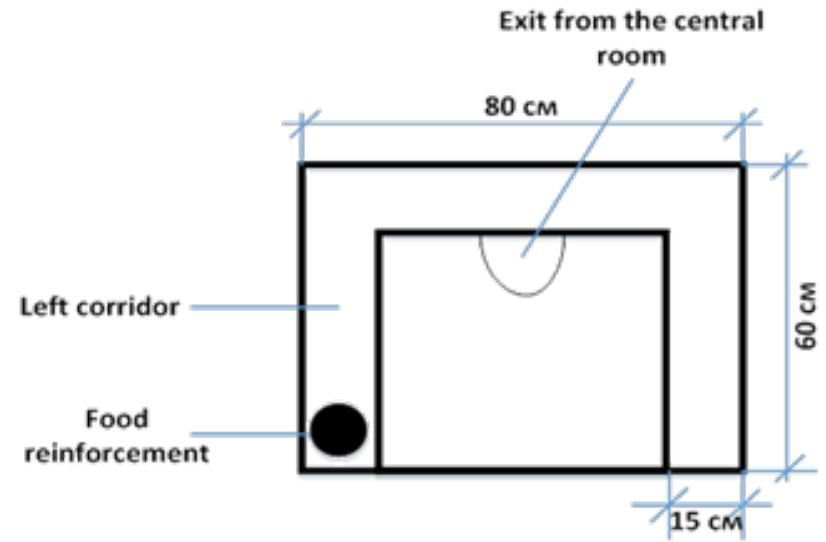

Figure I The picture and the chart of T-shaped maze for the study of food conditional reflex.

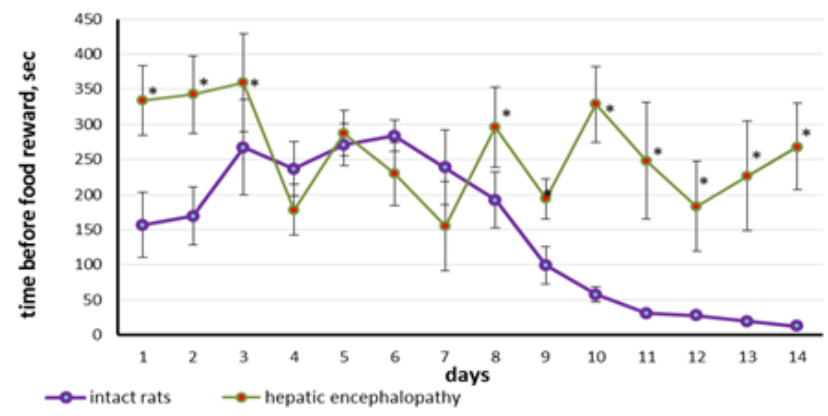

Figure 2 Time before food reward at the development of food conditioned reflex in the rats with $\mathrm{CCl}_{4}$-induced cirrhosis and intact rats.
However, there were not registered data about faster food reinforcement in the group of animals that got $\mathrm{CCl}_{4}$ injection. Moreover, in the group of intact rats we have established the decrease in time of finding the food by $92 \%(\mathrm{p}<0.05)$ as compared with the first day of experiment (Figure 2). Such changes were not obvious for the $\mathrm{CCl}_{4}$-group: we did not find the significant difference between the reaching food of $\mathrm{CCl}_{4}$-rats at the start and at the 14th day of experiment in the T-shaped maze. Thus, we registered that the time of finding the food in the $\mathrm{CCl}_{4}$ group was $21.4(\mathrm{p}<0.001)$ greater than in the intact group at the 14th day of study in the T-shaped maze (Figure 3 ). The impairment of food conditioned reflex formation has showed lack of remembering and thinking in rats pretreated with $\mathrm{CCl}_{4}$. This confirms the progression of liver pathology and concomitant dystrophy of brain tissue with the development of HE.

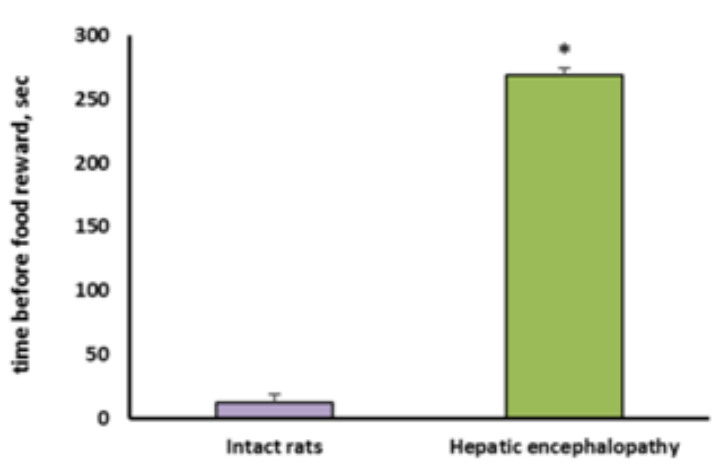

Figure 3 Time before food reward at the 14th day during the development of food conditioned reflex in the rats with $\mathrm{CCl}_{4}$-induced cirrhosis and intact rats $(\mathrm{M} \pm \mathrm{m})$.

${ }^{*} \mathrm{p}<0.05$ compasred with intact rats.

\section{Conclusion}

We have updated the scheme of modeling LC and HE by the decrease of time of the $\mathrm{LC}$ development and the reduction of animal mortality as result of toxic action by $\mathrm{CCl}_{4}$. The improved diagnosis of HE was achieved by using the methodology of forming a food conditioned reflex in rats. This method can be recommended as a basic for HE modeling and can be used for study new treatment strategies of this disease.

\section{Acknowledgements}

None.

\section{Conflict of interest}

Author declares that there is no conflict of interest.

\section{References}

1. Liu A, Perumpail RB, Kumari R, et al. Advances in cirrhosis: optimizing the management of hepatic encephalopathy. World J Hepatol. 2015;7(29):2871-2879.

2. Mendizabal M, Silva MO. Liver transplantation in acute liver failure: a challenging scenario. World J Gastroenterol. 2016;22(4):1523-1531.

3. Rahimi RS, Rockey DC. Hepatic encephalopathy: how to test and treat. Curr Opin Gastroenterol. 2014;30(3):265-271.

4. Wang JY, Zhang NP, Chi BR, et al. Prevalence of minimal hepatic encephalopathy and quality of life evaluations in hospitalized cirrhotic patients in china. World J Gastroenterol. 2013;19(30):4984-4991. 
5. Hepatic encephalopathy in chronic liver disease: 2014 practice guideline by the European Association for the study of the liver and the American Association for the study of liver diseases. $J$ Hepatol. 2014;61(3):642-659.

6. Grover VP, Tognarelli JM, Massie N, et al. The why and wherefore of hepatic encephalopathy. Int J Gen Med. 2015;8:381-390.
7. Huang YW, Yang SS, Kao JH. Pathogenesis and management of alcoholic liver cirrhosis: a review. Hepat Med. 2011;3:1-11.

8. Yang L, Wang Y, Wang X, et al. Effect of allogeneic umbilical cord mesenchymal stem cell transplantation in a rat model of hepatic cirrhosis. J Tradit Chin Med. 2015;35(1):63-68. 